\title{
ERROR COMPENSATION IN INSERTION-REGION MAGNETS
}

\author{
JIE WEI \\ Relativistic Heavy Ion Collider, Brookhaven National Laboratory, \\ Upton, New York 11973, USA
}

(Received 2 January 1996; in final form 2 January 1996)

\begin{abstract}
Methods used to compensate the effects of nonlinear field errors in the RHIC insertion-region
\end{abstract} magnets are discussed.

Keywords: Magnet design; correction scheme.

\section{INTRODUCTION}

In heavy ion storage rings, intra-beam scattering (IBS) between high charge state ions results in a large beam emittance during storage. The ultimate machine performance depends on achieving the highest possible magnetic field quality and alignment accuracy in the insertion-region (IR) triplet magnets during low- $\beta^{*}$ operation when the beam size is the largest in the triplets. Therefore, effective compensation of magnet construction errors and misalignments is crucial.

Heavy-ion beams $\left(\mathrm{Au}^{79+}\right)$ will be accelerated and stored for 10 hours in the Relativistic Heavy Ion Collider (RHIC) at the energy of $100 \mathrm{GeV} / \mathrm{u}$ in two separated rings consisting of superconducting magnets. Due to strong IBS, the transverse beam emittance grows from $10 \pi \mathrm{mm} \cdot \mathrm{mr}$ at injection to more than $40 \pi \mathrm{mm} \cdot \mathrm{mr}$ at storage. Dipoles and triplets of quadrupoles of large bore are placed on both sides of the six interaction points (IP). In order to maximize the luminosity at two IPs with proposed experiments, the nearby triplets are designed to enable the collision $\beta$-function to be reduced to $\beta^{*}=1 \mathrm{~m}$. Consequently, the rms transverse beam size becomes large at the triplets $\left(\beta_{\max }=1400 \mathrm{~m}\right.$ ), increasing from $\sigma=2.3 \mathrm{~mm}$ to $4.7 \mathrm{~mm}$ during the period of storage. At the end of storage, the $5 \sigma$ beam size becomes about $71 \%$ of the coil radius $(65 \mathrm{~mm})$. The goal of IR triplet error compensation is 
to ensure satisfactory magnetic field quality and beam long-term stability up to this $5 \sigma$ radius.

Unlike industrial-built arc-region magnets discussed elsewhere, ${ }^{1,2}$ IR magnets are built on site at the Brookhaven National Laboratory Magnet Division. With extensive construction and measurement experience and intimate contact with the RHIC Accelerator Physics (RAP) group, it is possible for our magnet builders to implement some of the most advanced and sophisticated techniques on these magnets, including body-ends compensation, individual error correction with tuning shims, sorting, and lumped multi-layer corrector packages as discussed in Section 2.

Figure 1 shows the layout of the IR triplet cryostat that contains the magnets of both rings, each consisting of a $100 \mathrm{~mm}$ coil inner-diameter (ID) dipole (D0) and three $130 \mathrm{~mm}$ coil ID triplet quadrupoles (Q1, Q2, and Q3). All the quadrupoles have the same coil and iron cross-section in the main body, and the same lead-end and return-end coil winding. Each of the three lumped correctors, labeled $\mathrm{C} 1, \mathrm{C} 2$, and $\mathrm{C} 3$, contains four correction coil layers from dipole $\left(a_{0}\right.$ or $\left.b_{0}\right)$ to dodecapole $\left(a_{5}\right.$ or $\left.b_{5}\right)$ harmonics.

The various superconducting magnets are assembled at room temperature into the common cryostat. Once the assembly is completed, further adjustment in relative alignment is difficult. Therefore, an accurate alignment as well as a comprehensive knowledge of the warm-cold transitions is crucial (Section 3). Experience with arc-region Corrector-Quadrupole-Sextupole (CQS) assembly is thus extremely valuable. Figure 2 shows the significant improvement of the CQS alignment accuracy during the production process, ${ }^{3}$ which is made possible by the quick feedback between the magnet builders and RAP.

Because of the separated ring design, magnetic coupling between the side-by-side magnets in the two rings is generally small, except in the triplet region when the triplet dipoles (D0s) closely reside in the common cryostat. Furthermore, since the beam emittance at storage is large, the beam-beam interaction (total tune shift of $\Delta v \approx 0.005$ from 6 IR) is insignificant.

\section{MAGNET CONSTRUCTION ERROR COMPENSATION}

The magnet body field is expressed in terms of the multipole series

$$
B_{y}+i B_{x}=B_{0}\left[1+10^{-4} \sum^{n}\left(b_{n}+i a_{n}\right)\left(\frac{x+i y}{R_{0}}\right)^{n}\right],
$$




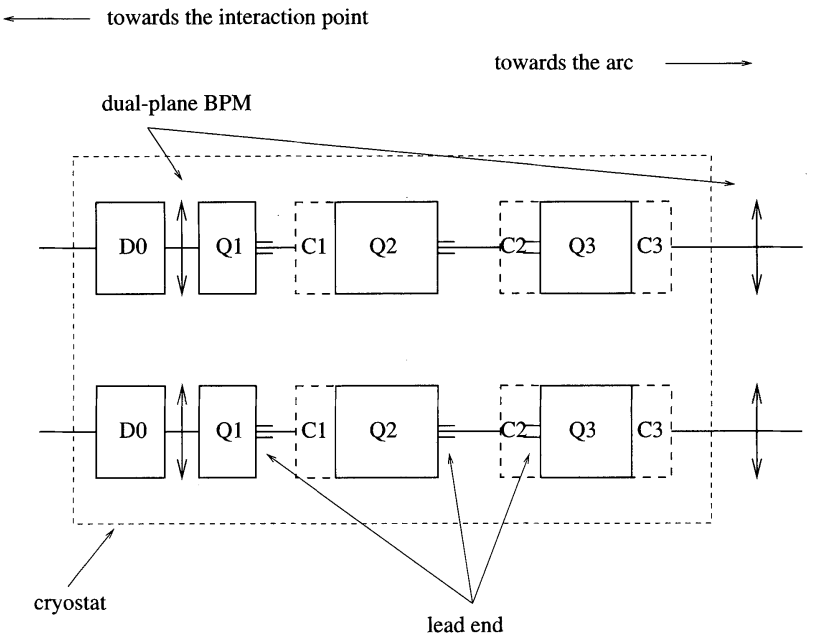

FIGURE 1 Schematic layout of the RHIC triplet cryostat assembly, showing the dipoles (D0), quadrupoles $(\mathrm{Q} 1, \mathrm{Q} 2$, and $\mathrm{Q} 3)$ and their lead-end orientation, triplet correctors $(\mathrm{C} 1, \mathrm{C} 2$, and C3), and dual-plane BPMs of both rings.

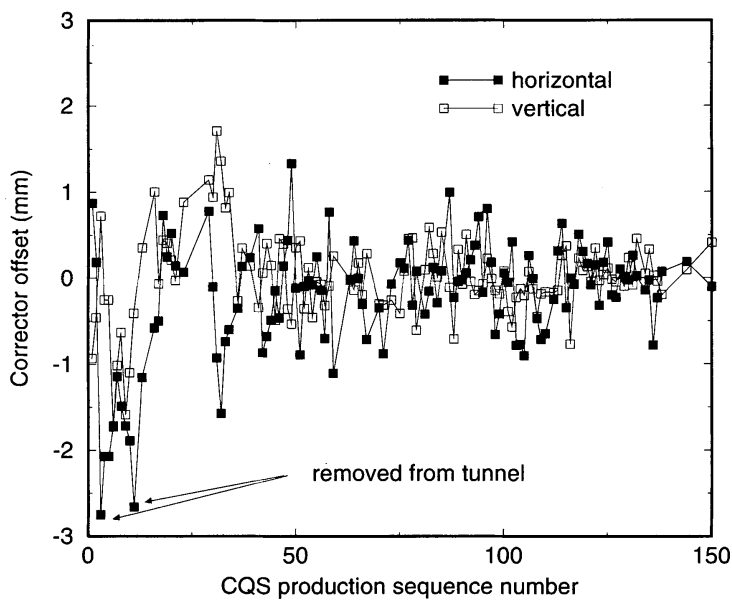

FIGURE 2 Trend plot of the corrector offsets from their ideal position in the CorrectorQuadrupole-Sextupole assemblies. The corrector offset is defined as the offset of the corrector center from its ideal beam trajectory with the quadrupole and sextupole perfectly aligned. The quadrupole center is determined by a colloidal-cell optical measurement, while the sextupole and corrector centers are determined by the mechanical measurement. 
TABLE I Expected values of the mean, uncertainty in mean, and standard deviation of the body, lead end, and return end harmonics of the triplet quadrupoles at storage $(5 \mathrm{kA})$, transition $(1.5 \mathrm{kA})$, and injection $(0.6 \mathrm{kA})$ current with nominal tuning shims inserted. If not specified, the value applies to all currents. The body harmonics $\left(b_{n}\right.$ and $\left.a_{n}\right)$ are expressed in $10^{-4}$ of the quadrupole body strength at the reference radius of $40 \mathrm{~mm}$. The lead-end and return-end harmonics are expressed in an integrated form in unit-meters

\begin{tabular}{|c|c|c|c|c|c|c|}
\hline Order, $n$ & & & Normal & & & Skew \\
\hline BODY & $\left\langle b_{n}\right\rangle$ & $d\left(b_{n}\right)$ & $\sigma\left(b_{n}\right)$ & $\left\langle a_{n}\right\rangle$ & $d\left(a_{n}\right)$ & $\sigma\left(a_{n}\right)$ \\
\hline 1 & 0.0 & 10.0 & 10.0 & 0.0 & 0.0 & 10.0 \\
\hline 2 & 0.0 & 0.5 & 2.4 & 0.0 & 0.1 & 1.2 \\
\hline 3 & 0.0 & 1.0 & 0.6 & 0.0 & 0.3 & 0.7 \\
\hline 4 & 0.0 & 0.3 & 0.6 & 0.0 & 0.1 & 0.5 \\
\hline $5(@ 5 \mathrm{kA})$ & -1.2 & 0.5 & 0.5 & 0.0 & 0.4 & 0.6 \\
\hline 7 & -0.2 & 0.1 & 0.1 & 0.0 & 0.0 & 0.1 \\
\hline $9(@ 5 \mathrm{kA})$ & 0.0 & 0.2 & 0.0 & 0.0 & 0.0 & 0.0 \\
\hline $5(@ 0.6 \mathrm{kA})$ & -4.7 & 0.5 & 0.5 & 0.0 & 0.4 & 0.6 \\
\hline $9(@ 0.6$ kA $)$ & -0.2 & 0.2 & 0.0 & 0.0 & 0.0 & 0.0 \\
\hline $5(@ 1.5 \mathrm{kA})$ & -2.2 & 0.5 & 0.5 & 0.0 & 0.4 & 0.6 \\
\hline $9(@ 1.5 \mathrm{kA})$ & -0.1 & 0.2 & 0.0 & 0.0 & 0.0 & 0.0 \\
\hline LEAD END & $\left\langle B_{n}\right\rangle$ & $d\left(B_{n}\right)$ & $\sigma\left(B_{n}\right)$ & $\left\langle A_{n}\right\rangle$ & $d\left(A_{n}\right)$ & $\sigma\left(A_{n}\right)$ \\
\hline 2 & 0.0 & 0.1 & 0.7 & 0.0 & 1.0 & 2.0 \\
\hline 3 & 0.0 & 0.3 & 0.3 & 0.0 & 0.4 & 0.8 \\
\hline 5 & 4.6 & 0.5 & 0.3 & -1.5 & 0.5 & 0.2 \\
\hline 9 & -0.5 & 0.1 & 0.0 & 0.2 & 0.1 & 0.0 \\
\hline RETURN END & $\left\langle B_{n}\right\rangle$ & $d\left(B_{n}\right)$ & $\sigma\left(B_{n}\right)$ & $\left\langle A_{n}\right\rangle$ & $d\left(A_{n}\right)$ & $\sigma\left(A_{n}\right)$ \\
\hline 2 & 0.0 & 0.3 & 1.8 & 0.0 & 0.7 & 1.0 \\
\hline 3 & 0.0 & 0.1 & 0.2 & 0.0 & 0.1 & 0.3 \\
\hline 5 & 1.0 & 0.0 & 0.6 & 0.0 & 0.1 & 0.1 \\
\hline 9 & -0.1 & 0.0 & 0.0 & 0.0 & 0.0 & 0.0 \\
\hline
\end{tabular}

defined at the reference radius $R_{0}$ of $5 / 8$ coil radius. For the triplet quadrupoles, $B_{0} \equiv G R_{0}$, where the nominal gradient $G=47 \mathrm{~T} / \mathrm{m}$, and $R_{0}=40 \mathrm{~mm}$.

A detailed description of the triplet quadrupole design and construction can be found elsewhere. ${ }^{4}$ The leading sources of un-desired harmonics are the design and construction errors in the placement of the coils at the ends and body of the magnets, e.g. large systematic $b_{5}$ and $a_{5}$ in the quadrupole lead ends, systematic $b_{3}$ and $b_{5}$ and random $b_{2}$ in the quadrupole body, as shown in Table II. The secondary source is the mislocation of the iron yoke, 
TABLE II The IR triplet correction strategy

\begin{tabular}{cll}
\hline Order, $n$ & Normal, $b_{n}$ & Skew, $a_{n}$ \\
\hline 0 & $\mathrm{C} 1$ or C3 & $\mathrm{C} 3$ or C1 \\
1 & individually powered & $\mathrm{C} 2$ \\
2 & $\mathrm{~S}, \mathrm{C} 3$ & $\mathrm{~S}, \mathrm{C} 2$ \\
3 & $\mathrm{~B}, \mathrm{~S}, \mathrm{C} 1, \mathrm{C} 3$ & $\mathrm{~S}, \mathrm{C} 2$ \\
4 & $\mathrm{~S}, \mathrm{C} 1$ & $\mathrm{~S}$ \\
5 & $\mathrm{~B}+, \mathrm{S}+, \mathrm{C} 1, \mathrm{C} 3$ & $\mathrm{~S}, \mathrm{C} 2$ \\
7 & $\mathrm{~B}$ & \\
9 & $\mathrm{~B}$ & \\
\hline
\end{tabular}

B: coil cross-section iteration

$\mathrm{B}+$ : coil cross-section iteration plus body-ends compensation

S: using tuning shims

$\mathrm{S}+$ : using tuning shims on random $b_{5}$ after body-ends compensation.

$\mathrm{C} 1, \mathrm{C} 2, \mathrm{C} 3$ : correction available at $\mathrm{C} 1, \mathrm{C} 2$, or $\mathrm{C} 3$ corrector

which results in excitation dependent $b_{5}$ and $b_{9}$ in the quadrupole body. Magnetic coupling between the magnets of the two rings is only significant for the D0 dipoles, resulting in effective $b_{1}, b_{3}$, etc. of about 0.5 units at the D0 reference radius of $R_{0}=31 \mathrm{~mm}$. Table II shows the expected values ${ }^{5}$ of the mean, uncertainty in mean, and standard deviation of some significant normal and skew quadrupole harmonics at various excitation currents generated in 1993. Recent warm and cold measurements of some fully and partially assembled triplet quadrupoles are in full agreement with Table II. It should be noted that only transverse components of the fringe field are listed in Table II. The effect of the longitudinal component of the fringe field has been shown to be insignificant. ${ }^{6}$

The betatron phase advance is negligibly small across D0 and the triplet, while the variation in transverse beam size is large from magnet body to end, and across the triplet. Therefore, error compensation of an un-desired multipole $b_{n}$ or $a_{n}$ is based on minimizing the total action kick in both horizontal and vertical directions across each triplet taking into account the variation of the design $\beta$-function,

$$
\int_{\text {triplet }} b_{n} \beta_{x, y}^{(n+1) / 2} d s \longrightarrow 0, \quad \int_{\text {triplet }} a_{n} \beta_{x, y}^{(n+1) / 2} d s \longrightarrow 0
$$


where the integral extends over all the quadrupole and D0 body and ends in one triplet.

Multipole optimization and body-ends compensation: The body coil cross section of the IR quadrupole has been iterated to minimize $b_{3}, b_{7}$, and $b_{9}$ in the body, and to compensate the $b_{5}$ from the ends. The yoke has been designed so that its saturation helps to optimize $b_{5}$ and $b_{9}$ at storage. Based on Equation 2, the body $b_{5}$ in all the triplet quadrupoles is chosen to be

$$
\begin{aligned}
b_{5}(\text { body }) & =-0.17\left[\mathrm{~m}^{-1}\right] B_{5}(\text { lead end })-0.35\left[\mathrm{~m}^{-1}\right] B_{5}(\text { return end }) \\
& =-0.12[\text { unit }]
\end{aligned}
$$

where the smaller coefficient for the lead end results from the optimization of the orientation of the lead ends. With this choice (Equation 3), the action kick given by $b_{5}$ is minimized for all low- $\beta^{*}$ operations in both horizontal and vertical directions.

Shimming: Tuning shims are planned to be inserted into the 8 empty slots of the IR quadrupole body after the magnet is constructed and individually warm measured. As shown in Table II, the variable thickness of these 8 shims enables the individual correction of 8 harmonics, from $a_{2}$ to $a_{5}$ and from $b_{2}$ to $b_{5}$, in the magnet body to their ideal values. Recent experiments with the tuning shims indicate that the thickness can be determined to a relative accuracy of about $10 \% \mathrm{rms}^{4}$

Sorting: The requirement on the field quality of the IR magnets becomes stringent only when $\beta^{*}$ is lowered near $1 \mathrm{~m}$. Since only two of the six interaction points are planned to operate at $\beta^{*}=1 \mathrm{~m}, 8$ "golden" magnets of each type will be selected for installation in the nearby triplets. These golden magnets will be evaluated and sorted after they are individually built and measured.

Local correctors: Lumped correctors located in the triplet region are used to correct closed-orbit errors, to perform local decoupling, and to correct higher-order multipole errors both from D0s and IR quadrupoles. Every triplet includes three corrector packages, $\mathrm{C} 1, \mathrm{C} 2$, and $\mathrm{C} 3$, each containing four layers of individually powered correction coils of various multipole type from normal and skew dipole $\left(b_{0} / a_{0}\right)$ to dodecapole $\left(b_{5}\right)$.

Because the $\beta$-function varies rapidly from body to end in the the triplet, higher-order multipole corrections in both horizontal and vertical directions (Equation 2) can be best achieved with two correctors in the triplet which 
are located at places with significantly different $\beta_{x} / \beta_{y}$ ratio. As shown in Table II, the $b_{3}$ and $b_{5}$ correctors designed to compensate for the residual error in the quadrupoles after shimming and for D0 cross coupling, are located at $\mathrm{C} 1$ and $\mathrm{C} 3$ where the ratio $\beta_{x} / \beta_{y}$ is large in one and small in the other. The excitation strength of these correctors is planned to be "dead-reckoned" based upon the cold multipole measurement of the IR quadrupoles and D0s, and the cold-measured integral transfer function of the correctors. Recently, "live" correction schemes are also proposed to adjust the corrector strengths according to the beam measurements. ${ }^{7}$

Every triplet contains a $b_{2}$ and a $b_{4}$ correctors at $\mathrm{C} 3$ and $\mathrm{C} 1$, respectively. All the skew correctors $a_{1}, a_{2}, a_{3}$, and $a_{5}$ are located at $\mathrm{C} 2$, where $\beta_{x}$ and $\beta_{y}$ are about equal. Except for $a_{1}$, all of these correctors are planned to be dead reckoned to correct the average construction error. The $a_{1}$ correctors, totally 12 per ring, are planned to be used ${ }^{8}$ along with the dual-plane Beam Position Monitors (BPMs) (Figure 1) for local decoupling.

\section{MAGNET MISALIGNMENT COMPENSATION}

Similar to the requirements on the construction error, the requirements on magnet misalignment become stringent during low- $\beta^{*}$ storage when the beam size at the triplet is large. The alignment procedure for the triplet assembly consists of several complicated steps including aligning the various corrector layers, the corrector with respect to the quadrupole, the dipoles and quadrupoles of both rings with respect to each other, and the entire assembly as a rigid body (Figure 1). Because of the high $\beta$-function and their longer length, quadrupoles Q2 and Q3 are most sensitive to the misalignments. The accumulative errors from each step together with the complication caused by warm-cold transition make it a challenging task to achieve an accurate alignment.

Table III shows the effects of given final transverse offsets or rolls of the magnet, and the amount of correction needed. Effective closed-orbit correction using the triplet dipole correctors, which are highly effective due to their high $\beta$ locations, is essential to the successful operation during storage. Fortunately, since the betatron phase advance is small across the triplet region, it is possible to use the normal and skew dipole correctors to correct the closed orbit error produced by the misalignment of the magnets in the triplet. On the other hand, since a given undesired beam observable may 
TABLE III Effects of a transverse offset or roll of the magnet in the triplet assembly from its design beam trajectory. When not specified, it is assumed that $\beta^{*}=1 \mathrm{~m}$ at storage with operating current of $5 \mathrm{kA}$

\begin{tabular}{llll}
\hline Magnet & $\begin{array}{l}\text { Offset } \\
\text { or roll }\end{array}$ & $\begin{array}{l}\text { Effects for } \beta^{*}=1 \text { m operation } \\
\left.\text { (for } \beta^{*}=2 \text { m operation }\right)\end{array}$ & $\begin{array}{l}\text { Corrector } \\
\text { needed }\end{array}$ \\
\hline D0 & $0.5 \mathrm{~mm}$ & $\begin{array}{l}\text { aperture reduction of } 8 \% \text { ms beam radius } \\
\left(11 \% \text { rms beam radius at } \beta^{*}=2 \mathrm{~m}\right)\end{array}$ & \\
& $0.5 \mathrm{mr}$ & $\begin{array}{l}\text { closed orbit offset of } 1.3 \mathrm{~mm} \text { at arc } \\
\left(0.9 \mathrm{~mm} \text { at } \beta^{*}=2 \mathrm{~m}\right) \\
\text { Qlosed orbit offset of } 23 \mathrm{~mm} \text { at arc }\end{array}$ & $a_{0} / b_{0}$ at $1 \mathrm{~A}$ \\
& $0.5 \mathrm{~mm}$ & $\begin{array}{l}\left(16 \mathrm{~mm} \text { if } \beta^{*}=2 \mathrm{~m}\right) \\
\text { equivalent to } \Delta Q_{\min }=0.026^{b} \\
\left(\Delta Q_{\min }=0.013 \text { at } \beta^{*}=2 \mathrm{~m}\right)\end{array}$ & $a_{1}$ at $7 \mathrm{~A}$ \\
& $0.5 \mathrm{mr}$ &
\end{tabular}

(a) At arc section with $\beta=50 \mathrm{~m}$.

(b) Minimum tune split due to linear coupling.

have many origins, correcting for the misalignments will be complex in the case of closed orbit errors, especially when the entire triplet is corrected for each transverse direction by only one corrector whose maximum current is $50 \mathrm{~A}$. It is therefore essential to limit the offset of each individual triplet dipole, quadrupole, and corrector to be as small as reasonably achievable.

During assembly of the arc-region CQS package, the welding is choreographed to balance distortion and to minimize offset. A colloidal cell optical technique has also been successfully used to locate the magnetic center of the quadrupole relative to external fiducials, after the magnet is fully assembled in the CQS package. These techniques will be further developed and adopted for the triplet assembly.

\section{COMPUTER MODELLING}

Computer modelling of the various correction steps has been performed mostly using ${ }^{9}$ program TEAPOT along with the TEAPOT "filter" TUNEIR that we newly developed. ${ }^{10}$ This filter program reads the standard TEAPOT machine file that contains magnet multipoles, simulates various compensation steps, produces tune footprints, and records output into an updated TEAPOT multipole file for further tune footprint and dynamic 


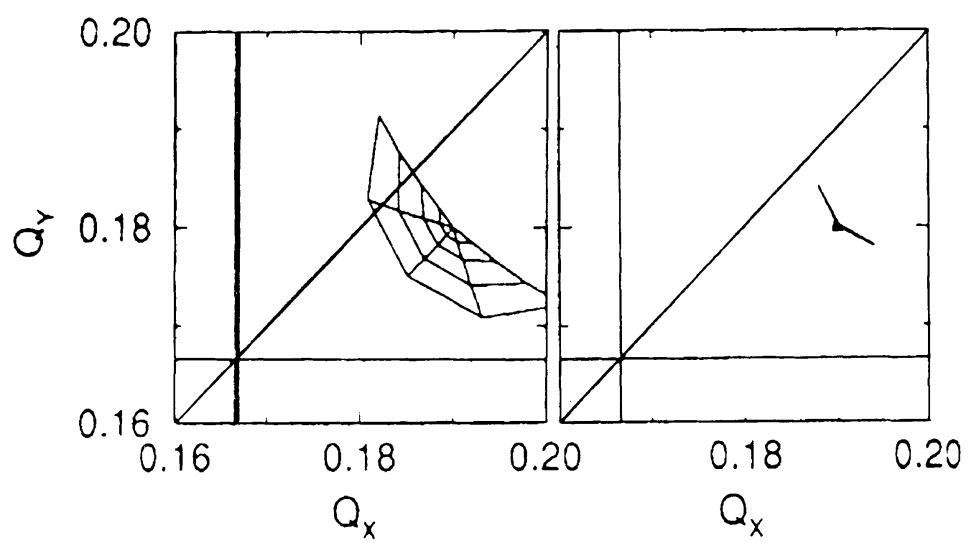

FIGURE 3 The tune footprint before and after the triplet correction using shimming and local correctors.

aperture analysis. Figure 3 shows the $5 \sigma$ tune footprints before and after the compensation produced by TUNE-IR. The rms measurement error of the multipoles has been assumed to be $10 \%$. The correction is very effective in reducing the spread of the footprint to a level that is negligible compared to the chromatic spread. After orbit correction, local decoupling, and triplet local correction, the dynamic aperture ${ }^{10}$ determined by 100 synchrotron periods of tracking is about $5 \sigma$.

\section{CONCLUSIONS}

With a comprehensive knowledge of magnet warm-cold correlation and fringe field, a four-step procedure for IR triplet error compensation has been adopted consisting of body-ends compensation, shimming, sorting, and using local correctors. The local triplet correctors are essential in eliminating construction and alignment errors.

\section{Acknowledgements}

I would like to thank S. Peggs and R. Gupta for their collaborative contribution to this work, and A. Jain, D. Trbojevic, P. Wanderer, M. Harrison and many other Magnet Division friends for the help and discussions. 


\section{References}

[1] S. Peggs, Feedback between Accelerator Physicists and Magnet Builders, these Proceedings.

[2] J. Wei, R.C. Gupta, A. Jain, S.G. Peggs, C.G. Trahern, D. Trbojevic and P. Wanderer, Field Quality Evaluation of the Superconducting Magnets of the Relativistic Heavy Ion Collider, Proc. 1995 Part. Accel. Confe., Dallas, Texas, (1995), (to be published).

[3] J. Wei, S. Peggs, S. Tepikian, P.A. Thompson and D. Trbojevic, Effects of CQS and Dipole Misalignments in RHIC, RHIC/AP/71 (1995).

[4] R. Gupta, Estimating and Adjusting Field Quality in Superconducting Magnets, these proceedings.

[5] J. Wei, R. Gupta and S. Peggs, Magnet Correction of RHIC Triplets, Proc. 1993 Part. Accel. Confe., Washington, D.C., (1993), p. 257.

[6] J. Wei and R. Talman, Theorem on Magnet Fringe Field, these Proceedings.

[7] For example, V. Ziemann, Measuring Hamiltonian Coefficients with a Wobbling Method, these Proceedings, and R. Talman (unpublished).

[8] F. Pilat, S. Peggs, S. Tepikian, D. Trbojevic and J. Wei, The Effect and Correction of Coupling Generated by the RHIC Triplet Quadrupoles, Proc. 1995 Part. Accel. Confe., Dallas, Texas, (1995), (to be published).

[9] L. Schachinger and R. Talman, "TEAPOT, Thin Element Tracking Program for Optics and Tracking", Particle Accelerators, 1987.

[10] J. Wei, G.F. Dell, S. Peggs, T. Satogata and S. Tepikian, Comparison of Perturbation Method and TEAPOT Tracking on Tune Shift Calculations, RHIC/AP5, (1993). 